\title{
Research on Organization and Performance of High Carbon Forging Bainite Wear-resistant Steel
}

\author{
Penghui Yang ${ }^{1, a}$, Shoufan Rong ${ }^{1,}{ }^{*}$, Yongchang Zhu1 ${ }^{1}$, Yanhe $\mathrm{Wu}^{1}$, Xiulan \\ Duan ${ }^{1}$, LiuHui ${ }^{1}$, Yunzhao Ding ${ }^{1}$ \\ ${ }^{1}$ School of Materials Science and Engineering, Jiamusi University, Jiamusi, 154007, China \\ ag87910162@qq.com \\ * Corresponding author's email: rsfzz@163.com
}

\begin{abstract}
Keywords: Grinding ball; Forging; High carbon wear resistant steel; Organization performance Abstract. According to the phenomenon of the grinding ball wide application and low life, put forward on the basis of high carbon wear resistant steel and then optimized composition, adopted the forging and the isothermal quenching process to improve the organization and performance so as to improve the service life of the grinding ball in this paper. Founding a set of comprehensive mechanical properties chemical composition and heat treatment process through the analysis and discussion of experimental results. Compared with the steel forging experiments, the average hardness of experiment steel is increased by $1 \mathrm{HRC}$ and impact toughness increased by more than 7 $\mathrm{J} / \mathrm{cm}^{2}$.
\end{abstract}

\section{Introduction}

Grinding ball [1-5] is widely used in cement, mining, thermal power, magnetic materials and other non-metallic powder processing and engineering, it's important parts of a ball mill. There is a low hardness, hardness uneven distribution process easy to wear and cracking capacity when using high balls and other issues in the past production process. The product of the ball is not good in use effect, it's far short of international standards. They caused a lot of waste of raw materials, increased production costs, bringing unnecessary economic losses.

In this paper, According to a series of problems and shortcomings exists in ball, high-carbon steel forging wear studies were analyzed from ingredient in many ways and technology. Ultimately, the ideal ball high life "within the tough outer hard 'performance were obtained by use of computer simulation, optimization of chemical composition, improved process.

\section{Experimental Materials and Methods}

Chemical composition. Effect of Mechanical Properties of Carbon Steel Casting are many factors that determine the performance the most basic, the most important internal factor is the chemical composition. Change the chemical composition can be effective in changing the organization and performance of steel.

Carbon as the main strengthening element of the steel matrix organization play a decisive role, a small amount of carbon dissolved in ferrite, the rest existed in the form of cementite carbon. Research carbon impact on high-carbon forged bainitic steel by changing the experimental carbon content in steel, its chemical composition as shown in Table 2.1.

Chromium increased bainite incubation period. With increasing chromium content, pearlite transformation part slightly move to the high temperature and the bainite move to the low temperature. It is improve the strength, hardness while also improving its plasticity and toughness when the chromium content is less than $2 \%$. Therefore, the design of the chromium content of $2 \%$.

The main role of manganese is deoxygenated and neutralize the harmful effects of sulfur, prevent the casting hot cracking and other defects. Manganese in steel can significantly improve the steel harden-ability, make the whole TTT curve shifts to the right, while the manganese on the substrate as well as some solid solution strengthening effect. 
Silicon is a useful element in the steel, its main role is to make liquid steel deoxidation. While silicon can strengthen ferrite, improve strength and yield ratio.

Molybdenum solid solution form in the steel, but also the formation of carbides, molybdenum can significantly improve harden-ability, the steel can be obtained in a continuous cooling bainite, it is a major element in the bainitic steel.

The above influence of alloying elements on bainitic steel, the paper identified the chemical composition as shown in Table 1.

Table 1 Experimental steel chemical composition table

\begin{tabular}{cccccc}
\hline & $\mathrm{C}$ & $\mathrm{Cr}$ & $\mathrm{Mn}$ & $\mathrm{Si}$ & Mo \\
\hline 1 & 0.75 & 2 & 2 & 1.5 & 0.3 \\
2 & 0.85 & 2 & 2 & 1.5 & 0.3 \\
3 & 0.95 & 2 & 2 & 1.5 & 0.3 \\
4 & 1.05 & 2 & 2 & 1.5 & 0.3 \\
\hline
\end{tabular}

Process scheme. Accurate dosing according to the design of the steel component. Added sequentially pig iron, scrap steel, high carbon ferrochrome, high-carbon ferromanganese, ferrosilicon and other ferroalloys respectly, aluminum wire prior to pouring sufficiently deoxygenated and adding small amounts of Rare Earth Modification. Steel using "high-temperature furnace, low temperature pouring", tapping temperature controlled at about $1550{ }^{\circ} \mathrm{C}$., pouring temperature control in $1480 \sim 1500{ }^{\circ} \mathrm{C}$. Forging relative deformation amount determined to be $60 \%$, simulation out TTT curve and CCT curve by the use of computer, determine isothermal hardening heat treatment process parameters.

In this paper, the desired sample were: $10 \mathrm{~mm} \times 10 \mathrm{~mm} \times 55 \mathrm{~mm}$ standard impact test sample; $15 \mathrm{~mm} \times 150 \mathrm{~mm} \times 40 \mathrm{~mm}$ forged specimen; a $120 \mathrm{~mm}$ diameter ball specimens.

Test and inspection equipment. Using the GPS-800 type $50 \mathrm{~kg}$ IF furnace smelt alloy steel, RX-45-9 box-type heat treatment furnace; impact and hardness were test by useed ZBC-300B automatic impact testing machine and $\mathrm{R}$ (D) -150A1 Rockwell hardness tester; metallographic analied by used an optical microscope Olympus GX71: Forged tested by used 500kg air hammer.

\section{Experimental results and analysis}

Heat treatment processes. In order to study the isothermal forging time bainite Steel properties of law, this article will isothermal time was set at $1 \mathrm{~h}, 2 \mathrm{~h}, 3 \mathrm{~h}$. Heat treatment process curve shown in Figure 1 finally.

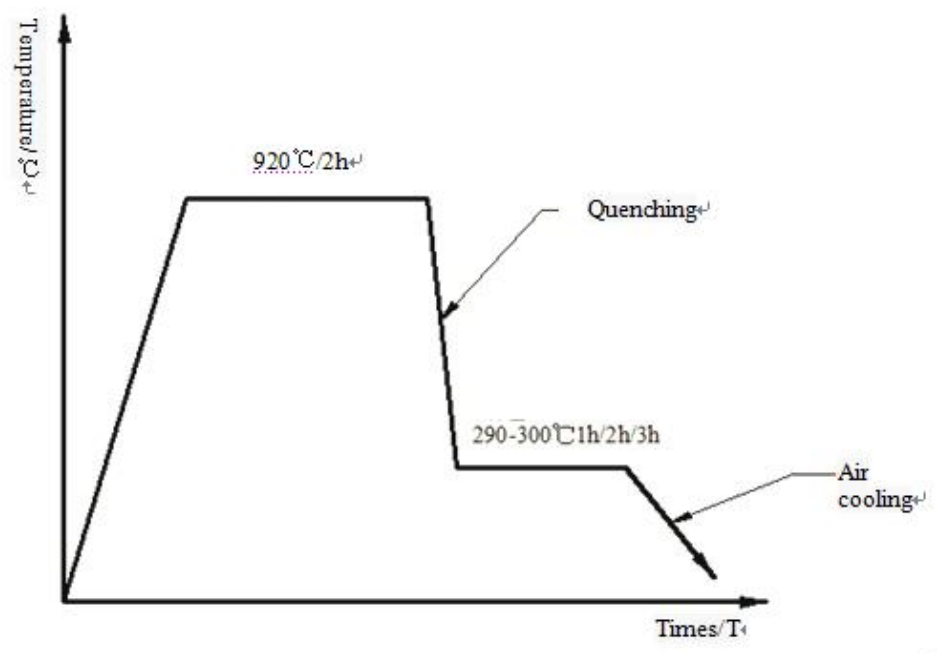

Figure 1 Graph Heat Treatment

Effect of carbon content on the microstructure and properties. The carbon content of steel used in this paper were higher than outside in addition to $0.75 \%$ eutectoid point, this kind of steel in the heat treatment process, starting precipitate cementite from austenite, this proeutectoid distributed along the austenite cementite of grain boundaries, acicular distributed within the crystal when much. Because of the presence of such organizations in the hard carbide, significantly higher material 
hardness and grain boundary carbides have split the role of the matrix such that toughness decreases significantly, is not conducive to practical application, therefore, to improve high carbon steel toughness by forging in this article. High carbon content of different carbon phase diagram shown in Figure 2.

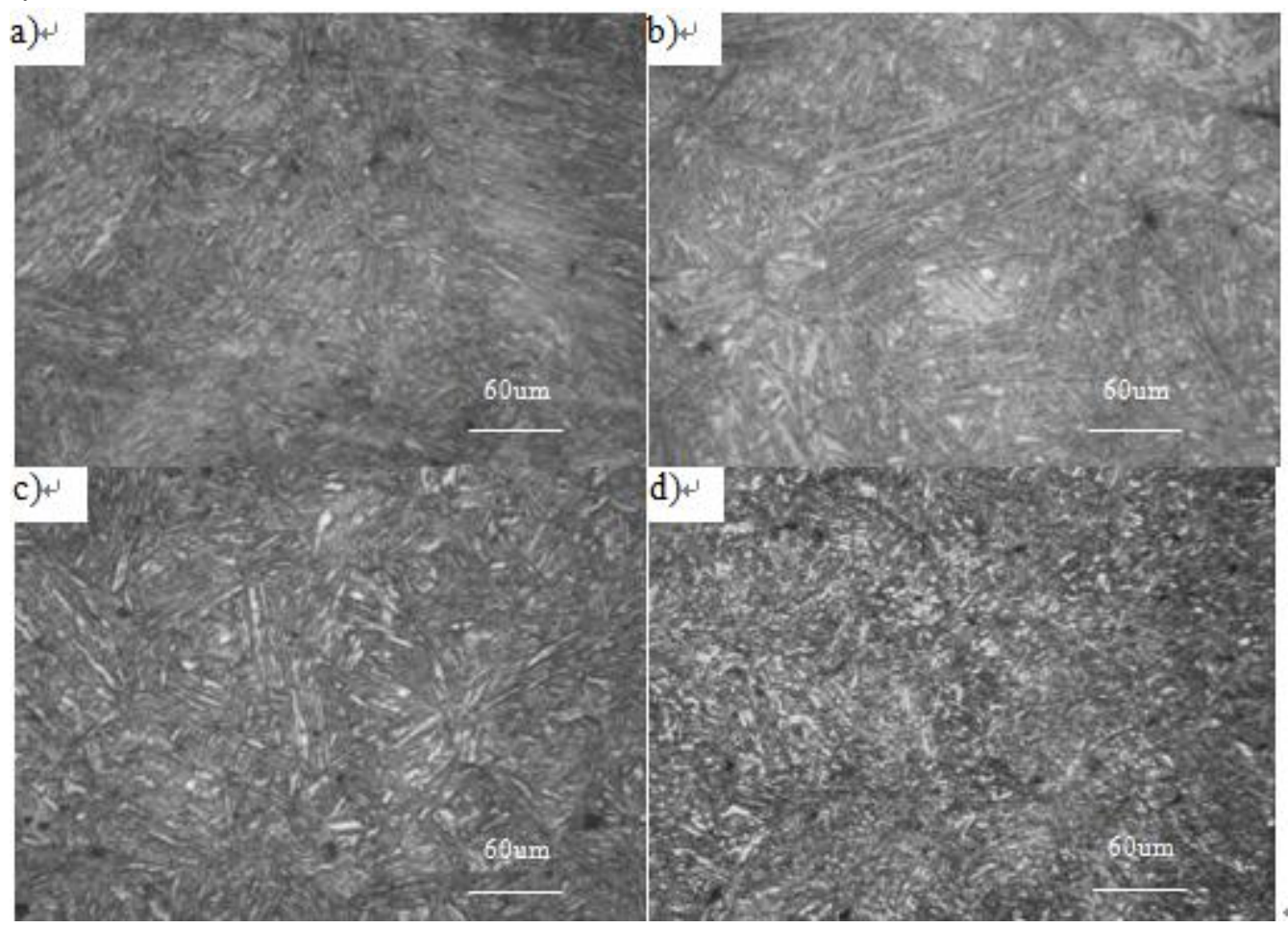

Figure 2 Different high carbon content steel microstructure Figure

a) the carbon content of $0.75 \%$; b) carbon content of $0.85 \%$;

c) the carbon content of $0.95 \%$; d) the carbon content of $1.05 \%$

Metallographic Figure 2 composed of the black portion, the white shiny section and white dark portion, the portion of white shiny is carbide, with the increase of carbon content of the sample, such white hair the organization gradually increased mostly bright or needle point from Fig $2 b$ to Fig 2d. Because of the increased amount of carbon increases the carbon atoms precipitate from the austenite amount, which increases the number of carbides. Increasing carbon content, making metallurgical took place larger organizational changes, also the mechanical properties of the apparent differences in the mechanical properties of the curve shown in Figure 3. Carbon content from $0.75 \%$ to $1.05 \%$, Rockwell hardness increased significantly, but the impact toughness actually was a clear downward trend, in which the carbon content of $1.05 \%$ of the high-carbon steel specimen toughness average of only $6 \mathrm{~J} / \mathrm{cm}^{2}$ or so. Carbide needle to split the role of the matrix is particularly strong, which is the main reasons to increase the carbon content of brittle material.

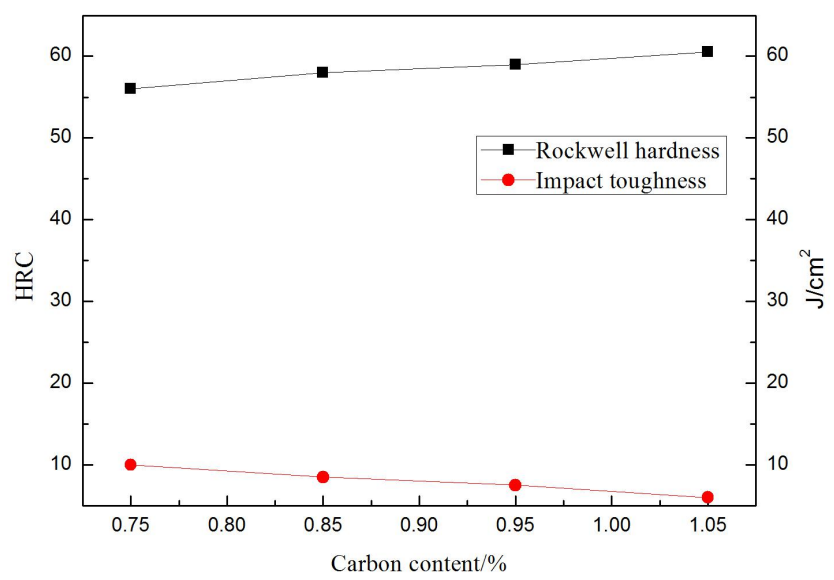

Figure 3 Different carbon content of high-carbon steel mechanical properties of graph 


\section{Effect of heat treatment on the microstructure and properties.}

Effect of heat treatment on hardenability. This article is a harden-ability test sample after cutting ball from the edge to the core section sequentially detects Rockwell hardness obtained. The test results shown in Figure 4, the distance shown in the figure is from the edge of the sample to the test point. As can be seen, the chemical composition for this article and heat treatment process are not designed to make balls sample is completely quenching, the carbon content of 1.05 percent and 0.95 percent of the ball harden-ability specimen from the edge when $4 \mathrm{~cm}$, ball hardness remains in 54HRC above. In practice used of the balls, the low hardness of heart can resist the impact of certain loads, ensure ball is not broken, surface hardness is high and good wear resistance. This performance of "inner tough outer hard" can effectively extend the life of the ball. Carbon content increasing, make the critical cooling rate of quenching is decreased, increase harden-ability, therefore, the carbon content $1.05 \%$ and $0.95 \%$ of the sample the permeable layer is very thick, away from the edge of $4 \mathrm{~cm}$, ball hardness is still high.

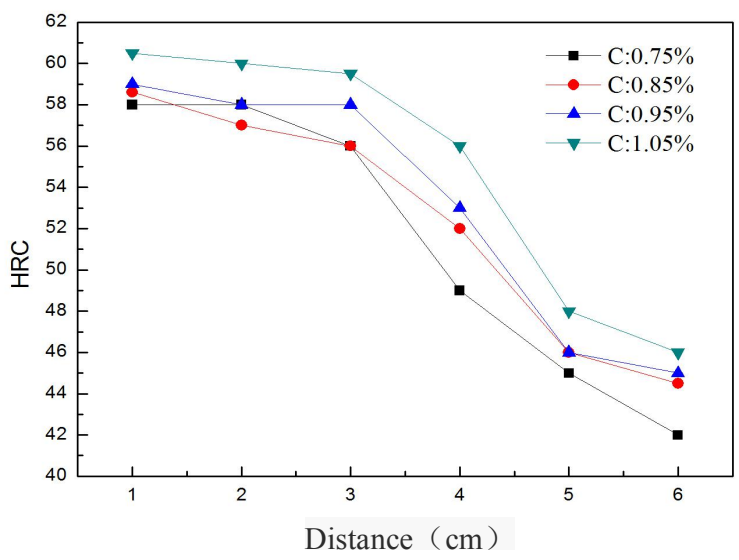

Figure 4 hardenability test results in Fig.

Influence of isothermal time on the microstructure and properties. Under the isothermal hardening temperature premise of $290 \quad{ }^{\circ} \mathrm{C}$. $\sim 300 \quad{ }^{\circ} \mathrm{C}$., the short holding time is not enough to get the next sample bainite and the super-cooled austenite directly into martensite point following form martensite. Figuer 5 is different isothermal time high carbon steel microstructure figure.

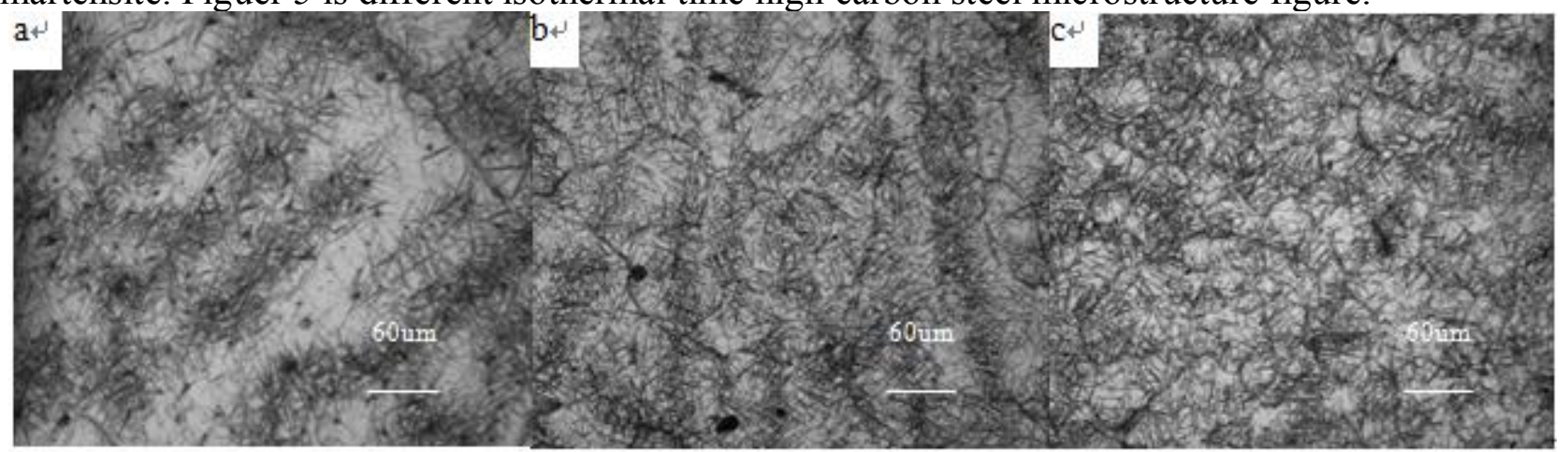

Figure 5 Different time isotherm high carbon steel microstructure Figure
a) $1 \mathrm{~h} ; \mathrm{b}) 2 \mathrm{~h} ; \mathrm{c}) 3 \mathrm{~h}$

As is shown from Figure 5a, when incubation time was 1h, High Carbon Steel with bamboo-like twin martensite main part of the retained austenite supplement. Figure $5 \mathrm{~b}$ and $5 \mathrm{c}$, the black and the semi-strip and the lens sheet organization for the next bainite, the analysis and calculation, from $b$ to c significantly increased the number of lower bainite. This phenomenon is due with the extension of the isothermal time, the bainite carbon atom diffusion process more fully, bainite formed more stable. Different time isotherm changed the high-carbon steel microstructure and mechanical properties of high carbon steel also changed, Figure6 is a high-carbon steel mechanical properties change over time isothermal curve. Rockwell hardness of high carbon steel holding time grows downward trend, impact toughness rise. 


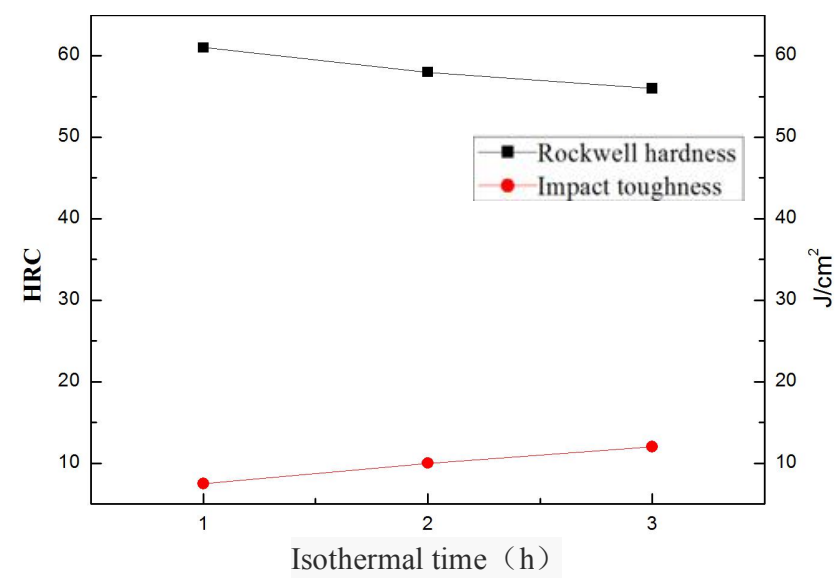

Figure 6 Different time isotherm mechanical properties of high carbon graph

Forging on Microstructure Properties. After forging the cast structure due to the deformation of the metal thermal processing method and recrystallization, so that the original coarse dendrite and columnar grains become finer grain, equiaxed recrystallized structure of uniform size, so that within the original ingot segregation, loose, pores, slag and other compaction and welding. After a one-way hot forging, microstructure and properties of high carbon steel has undergone great changes, figure 7 forging comparison chart and non forging high carbon steel microstructure.
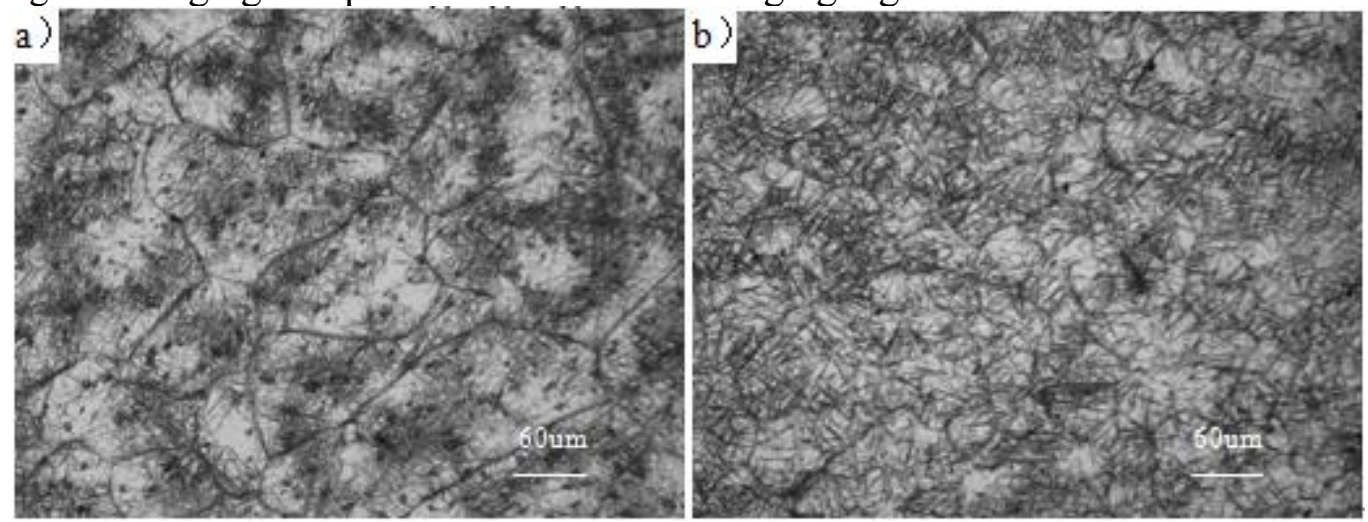

Figure 7 Comparison with non-forged high-carbon steel forging microstructure

a) Non-forging; b) forging

As can be seen from the microstructure after forging (Figure $7 b$ ), significantly higher than the non-grain forging forging fine, but after forging organizations bainite ferrite size thicker than non forging. After forged high-carbon steel, with the increase in the amount of deformation of the substrate distortion is getting worse, the base body of the dislocation density increasing, when the dislocation density of the metal rises to a certain value, the distortion to achieve a certain degree matrix deformation energy of internal storage and then place a sufficiently large dynamic recrystallization, grain reached a significant refinement. Since the forging temperature significantly higher than the non-heat treated forging austenitizing temperature, forging sample at high temperature austenite grain coarsening, and ultimately lead to the forging obtained bainitic ferrite size increases. Forging shown in Figure 8 with non-forged high-carbon steel mechanical properties of contrast curve. Compared with non-forged wrought specimens, defect reduction, compact, Rockwell hardness rose an average of about 1HRC. Carbon content between $0.75 \%$ to $0.95 \%$, after forging, impact toughness specimens significantly improved, but the carbon content of $1.05 \%$ of the forged specimens toughness lower than non-forged. Higher carbon content, secondary carbide precipitation at grain boundaries are more distributed, forging internal sample appeared microcracks, resulting in significantly lower toughness. 

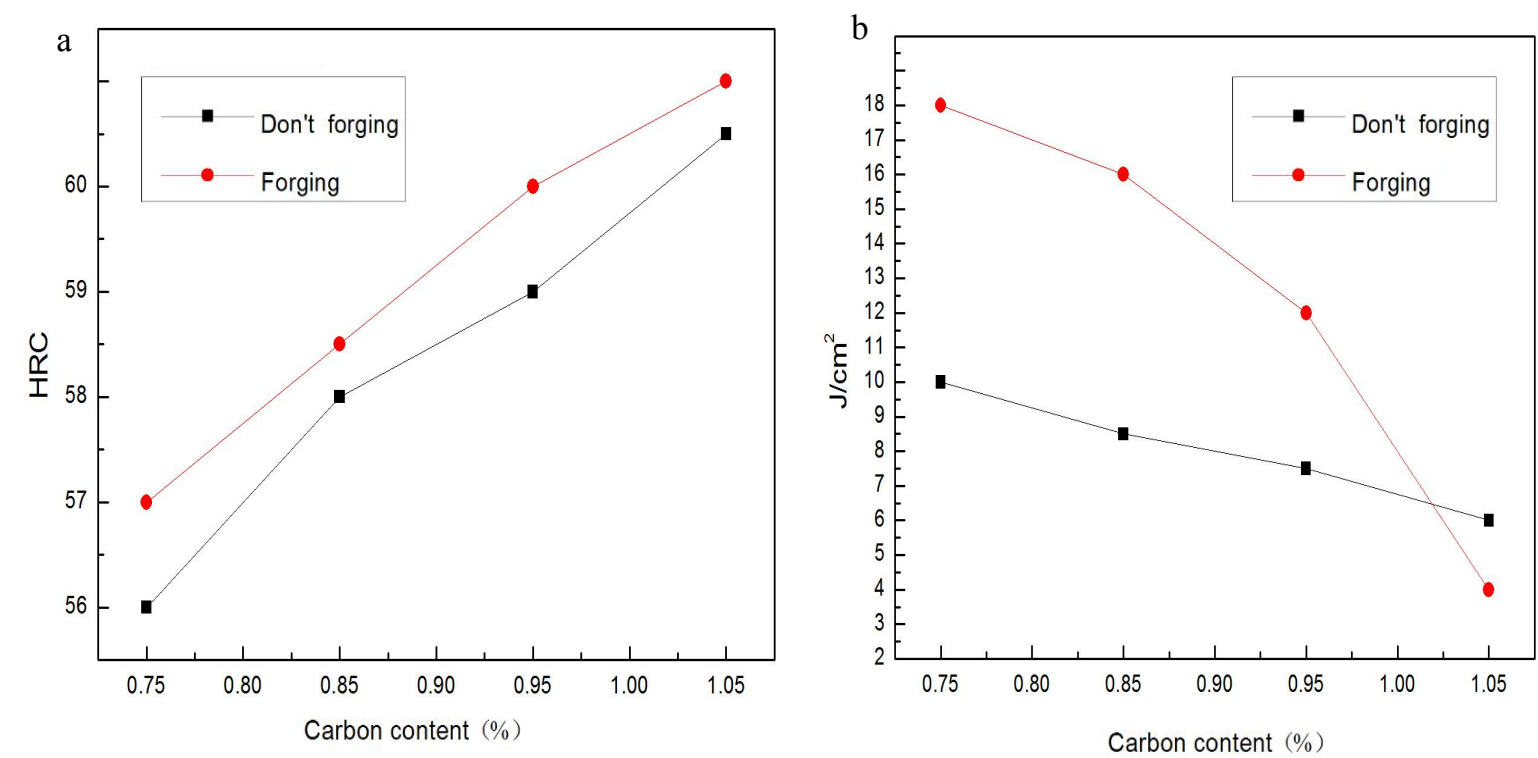

Figure 8 Non-forged high-carbon steel forging and mechanical properties of contrast curve

a) Non-forging; b) forging

\section{Conclusions}

Through computer simulation, process improvements and improved method of process parameters studied forged high-carbon bainitic microstructure and properties of steel, we obtained the following conclusions:

(1) The chemical composition (wt $\%$ ) of C: $0.95 \%$, Cr: $2 \%$, Mn: $2 \%$, Si: $1.5 \%$, Mo: 0.3 of forged high-carbon bainitic steel malleable nature, Rockwell hardness, integrated optimal toughness while access to over ball "inside tough outer hard 'performance.

(2) Chemical composition of high-carbon steel of conclusions 1, isothermal hardening time at least more than $2 \mathrm{~h}$ can get a stable lower bainite.

(3) When the carbon content below $0.95 \%$ the high-carbon steel forging make the experiment toughness improve average of $7 \mathrm{~J} / \mathrm{cm}^{2}$ or more, but continue to increase the carbon content of the microcracks occurred in the forging sample internal, resulting toughness low significantly.

\section{Acknowledgements}

This work is funded by the National Natural Science Foundation of China (Project No. 51371090), graduate science and technology innovation project (Project No. LZZ2015-005) Jiamusi University, Jiamusi University Innovation and Entrepreneurship Fund (Project No. xzyf2014-04).

\section{References}

[1] Hongming Lang. Production and selection of ball situation and development trend of [J]. Thermal processing technology. 2010-08-10

[2] Tiezhong Wang. Production and usage of our balls [J]. Water Resources and Electric Machinery. 2005-12-15

[3] S. C. Zanatta, F. F. Ivashita, K. L. Silva. Processing of gadolinium-iron garnet under non-equilibrium conditions[J]. Hyperfine Interactions, 2014, Vol.224 (1-3): .307-312

[4] K. N. Vdovin, I. V. Ponurko, M. V. Shubina. Technology for Making Partially Fusible Permanent Molds to Cast Iron Grinding Balls[J]. Metallurgist, 2014, Vol.45 (3), pp.167-170

[5] M. I. Timokhova. Study of certain factors in the wear of grinding balls during service[J]. Refractories and Industrial Ceramics, 2013, Vol.54 (1), pp.25-28. 